\title{
Truncation of geometrically thin disks around massive black holes in galactic nuclei
}

\author{
B. F. Liu ${ }^{1,2}$ and E. Meyer-Hofmeister ${ }^{1}$ \\ 1 Max-Planck-Institut für Astrophysik, Karl Schwarzschildstr. 1, 85740 Garching, Germany \\ 2 Yunnan Observatory, Academia Sinica. PO Box 110, Kunming 650011, PR China
}

Received 22 November 2000 / Accepted 16 March 2001

\begin{abstract}
The concept of an advection-dominated accretion flow (ADAF), with or without wind loss, was used to describe the spectra of the galactic center source $\mathrm{SgrA}^{*}$, low-luminosity AGN and nuclei of elliptical galaxies (including M 87). The spectral fits of various authors show that the transition from the geometrically thin disk to the hot flow occurs at quite different distances, apparently not uniquely related to the mass flow rate in the disk. We compare these results with the results of theoretical modeling where we determine the truncation of the thin outer disk from the efficiency of mass evaporation. The physics is the same as in the case of galactic black holes systems (Meyer et al. 2000b). For the observationally indicated mass flow rates our model predicts a truncation at $10^{3}$ to $10^{4}$ Schwarzschild radii. We discuss whether far inside this truncation an innermost cool thin disk could exist and affect the spectrum.
\end{abstract}

Key words. accretion disks - black hole physics - galaxies: nuclei - galaxies: individual: M 87, M 81 $\mathrm{X}$-rays: galaxies

\section{Introduction}

The form of accretion around black holes depends on the accretion rate. For low, sub-Eddington accretion rates a solution was found in which the accreting gas has a very low density and is unable to cool efficiently within an accretion time (Ichimaru 1977; Rees et al. 1982; Narayan \& Yi 1994, 1995a, 1995b; Abramowicz et al. 1995; Honma 1996; for a reviews see Narayan et al. 1998; Kato et al. 1998). In this mode of accretion (advection-dominated accretion flow, ADAF) advection rather than radiation removes the locally generated accretion heat. This is only possible in the inner region. At larger distance the mass accretes via a cool geometrically thin standard disk. Esin et al. (1997) considered accretion flows where the ADAF region extends outward to a certain transition radius, surrounded by a cool outside standard disk (with a corona above as continuation of the hot flow further inward). This picture was confirmed by the successful modeling of the spectral transitions observed for Nova Muscae 1991. In this investigation the transition was taken according to the "strong ADAF principle" (Narayan \& Yi 1995).

The transition to the ADAF can be understood as caused by evaporation of mass from the thin cool disk at the midplane to a hot coronal layer above. Such a corona

Send offprint requests to: E. Meyer-Hofmeister, e-mail: emm@mpa-garching.mpg.de exists predominantly above the inner region of the cool disk. The investigation of the equilibrium between these interacting layers shows that a continuous evaporation of gas occurs. The computation of the vertical structure of the corona allows to determine the amount of mass which flows inward in the corona at each distance from the black hole, depending on the mass of the compact star (Meyer \& Meyer-Hofmeister 1994). A detailed description of this process with application to disks around black holes is given in Meyer et al. (2000b). With decreasing distance from the black hole more and more mass flows in the corona. Where the coronal flow reaches $100 \%$ of the total accretion flow the cool disk in the midplane ends and inside is only a hot coronal/advection-dominated flow. The aim of the present paper is the application of our results to accretion disks around supermassive black holes.

Coronae in AGN were considered since many years by several authors (Liang \& Price 1977; Haardt \& Maraschi 1991; Nakamura \& Osaki 1993; Kusunose \& Mineshige 1994; Svensson \& Zdziarski 1994; Życki et al. 1995; Abramowicz et al. 1995 and subsequent workers). For a review on X-ray spectra of AGN see Mushotzky et al. (1993).

Recently the co-existence of the hot and the cold gas around galactic black holes and in AGN was investigated in detail by Różańska \& Czerny (2000a, 2000b). Their work follows in principle the same line as the work 
of Meyer \& Meyer-Hofmeister (1994) and Meyer et al. (2000b) on the equilibrium between corona and thin cool disk around a white dwarf or a black hole, but focuses on the innermost regions near the black hole and incorporates the physics of a two-temperature corona.

In new investigations of the low luminosity galactic nuclei of M 81 and NGC 4579 (Quataert et al. 1999) evidence was found for thin disks truncated at about 100 Schwarzschild radii, similar to the result for NGC 4258 (Gammie et al. 1999). The low luminosity in the elliptical galaxy M 87 (NGC 4486) was pointed out by Reynolds et al. (1996), illustrating the problem of a "quiescent" black hole. The nuclear regions of six elliptical galaxies were studied by Di Matteo et al. (1999, 2000). From the $\mathrm{X}$-ray emission together with recent high-frequency radio observations new constraints for the physical properties of any proposed ADAF were derived. These radio and submillimeter observations seem to demand that, at least in some galaxies, a significant fraction of mass, angular momentum and energy is removed from the accretion flow by a wind.

In this paper we first give a short description of the physics of evaporation, as included in our computations (Sect. 2). In Sect. 3 we present the results, and compare in Sect. 4 with findings from the spectra of low luminosity AGN and elliptical galaxies. In Sect. 5 we discuss a new suggestion for an innermost thin disk which comes from the evaporation/condensation picture and might help to solve discrepancies which were already found applying the ADAF model to low-luminosity galaxies.

\section{The equilibrium between thin disk below and corona above: Evaporation of cool gas}

The equilibrium between a cool disk and the corona above it is investigated in order to determine how much of the gas flows in the corona, that is, the amount evaporated from the cool disk into the corona. This evaporation efficiency strongly depends on the mass of the compact central object. For a given mass the efficiency increases with decreasing distance to the center (except in an innermost part). The co-existence of a cool disk and a corona had already been investigated for disks in dwarf nova systems (Meyer \& Meyer-Hofmeister 1994; Liu et al. 1995). The situation is the same in disks around neutron stars, stellar and supermassive black holes. For the properties of two-temperature, optically thin ADAFs (Narayan et al. 1998) successfully applied to accretion in stellar black holes and galactic nuclei, the quantities accretion rate and distance were written in units of Eddington accretion rate and Schwarzschild radius. $\left(\dot{M}_{\mathrm{Edd}}=40 \pi G M / \kappa c\right.$, $r_{\mathrm{S}}=2 G M / c^{2}$, with $M$ mass of central black hole and $\kappa$ electron scattering opacity). The solutions then are invariant. Our relation between evaporation rate and distance is also invariant.

We solve a set of four ordinary differential equations for motion of the gas, mass, energy and thermal flux (Meyer et al. 2000b) to model the equilibrium between cool disk and corona near the inner edge of the thin disk. The boundary conditions are: (1) at infinity no pressure (i.e. no artificial confinement, which requires sound transition at some height) and no influx of heat and (2) at the bottom of the corona chromosphere temperature and no heat inflow.

\section{Results of computations}

We carried out computations for massive black holes of $2.5 \times 10^{6}$ and $10^{8} M_{\odot}$ with Schwarzschild radii of $7.38 \times 10^{11}$ and $2.95 \times 10^{13} \mathrm{~cm}$, respectively. In Tables 1 and 2 we list mass flow rates $\dot{M}$ in the corona for a number of distances $r . \dot{M}$ and $r$ are also given in units of Eddington accretion rate and Schwarzschild radius. The relation between $\dot{M}$ and $r$ is invariant if accretion rates and distances are measured in these units. The same is true for the coronal temperature (compare Fig. 3 and the comments in Meyer et al. 2000b) and for the fraction of matter carried away by the wind. Also given are the values of pressure and mass flow rate into the corona at the lower boundary. Note that three similar rates are considered: the evaporation rate is equal to the mass flow rate in the thin disk at its inner edge; the mass flow rate in the corona is equal to the evaporation rate minus wind loss (accretion rate onto the black hole, if no wind loss further in).

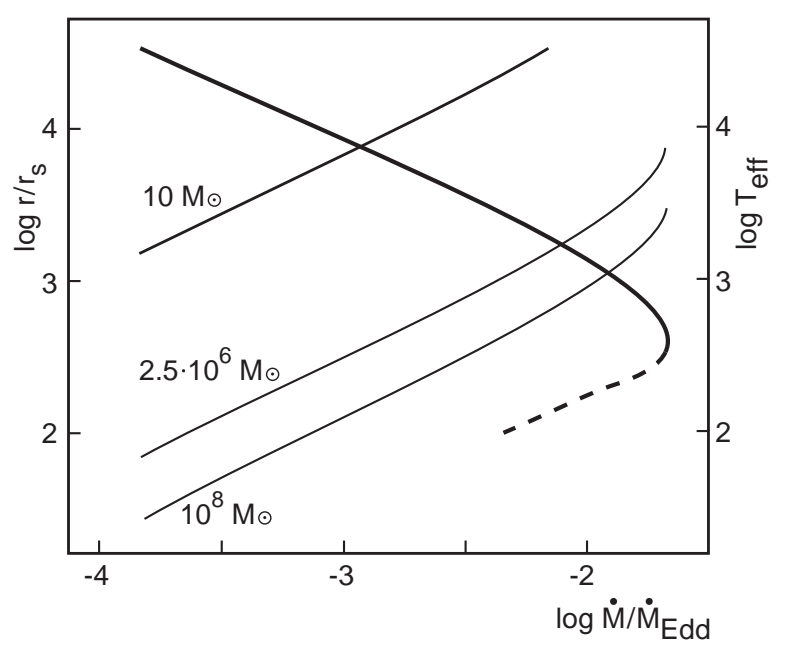

Fig. 1. Solid line: radius of transition from accretion via a geometrically thin disk to a coronal flow/ADAF (in units of Schwarzschild radius $r_{\mathrm{S}}$ ) as function of the mass flow rate in the corona (in units of the Eddington accretion rate $\dot{M}_{\text {Edd }}$ ), the dashed part of the relation is only an indication for a decreasing evaporation efficiency (reliable results require the consideration of a two-temperature corona). Thin lines: $T_{\text {eff }}$ effective temperature at the inner edge of the thin disk as function of radius $r_{\mathrm{S}}$ and accretion rate $\dot{M}$, (see text).

In Fig. 1 we show the relation between the location of the inner disk edge and the accretion rate. For example for the mass flow rate $\dot{M} / \dot{M}_{\text {Edd }}=10^{-3}$ the thin disk is truncated at about $6 \times 10^{3} \mathrm{Schwarzschild} \mathrm{radii.} \mathrm{Inside} \mathrm{the}$ 
Table 1. Evaporation of the disk around a black hole of $2.5 \times 10^{6} M_{\odot}$.

\begin{tabular}{cccccccc}
\hline $\log r$ & $\log \dot{m}_{0}$ & $\log P_{0}$ & $T(\mathrm{~K})$ & $\dot{M}=2 \pi r^{2} \dot{m}_{0}\left(M_{\odot} / \mathrm{yr}\right)$ & $\lambda$ & $\log r / r_{\mathrm{S}}$ & $\dot{M} / \dot{M}_{\mathrm{Edd}}$ \\
\hline 15.87 & -11.10 & -2.44 & $4.51 \times 10^{7}$ & $4.30 \times 10^{-5}$ & 0.23 & 4.00 & $7.81 \times 10^{-4}$ \\
15.48 & -9.79 & -0.93 & $1.10 \times 10^{8}$ & $1.47 \times 10^{-4}$ & 0.20 & 3.61 & $2.67 \times 10^{-3}$ \\
15.00 & -8.26 & 0.88 & $3.14 \times 10^{8}$ & $5.52 \times 10^{-4}$ & 0.15 & 3.13 & $1.00 \times 10^{-2}$ \\
14.60 & -7.17 & 2.26 & $6.85 \times 10^{8}$ & $1.09 \times 10^{-3}$ & 0.09 & 2.73 & $1.98 \times 10^{-2}$ \\
14.40 & -6.72 & 2.89 & $9.59 \times 10^{8}$ & $1.19 \times 10^{-3}$ & 0.04 & 2.53 & $2.16 \times 10^{-2}$ \\
14.30 & -6.57 & 3.14 & $1.06 \times 10^{9}$ & $1.07 \times 10^{-3}$ & 0.01 & 2.43 & $1.95 \times 10^{-2}$ \\
14.18 & -6.46 & 3.40 & $1.11 \times 10^{9}$ & $7.77 \times 10^{-4}$ & $\leq 10^{-3}$ & 2.31 & $1.41 \times 10^{-2}$
\end{tabular}

Notation: $\dot{m}_{0}$ and $P_{0}$ vertical mass flow density and pressure at the lower boundary of the corona, $T$ coronal temperature at the upper boundary (sound transition), $\dot{M}$ evaporation rate; $\lambda$ fraction of mass carried away by the wind; quantities $r / r_{\mathrm{S}}$ and $\dot{M} / \dot{M}_{\text {Edd }}$ scaled to Schwarzschild radius and Eddington accretion rate. $r, \dot{m}_{0}, P_{0}$ in cgs units.

Table 2. Evaporation of the disk around a black hole of $10^{8} M_{\odot}$.

\begin{tabular}{cccccccc}
\hline $\log r$ & $\log \dot{m}_{0}$ & $\log P_{0}$ & $T(\mathrm{~K})$ & $\dot{M}=2 \pi r^{2} \dot{m}_{0}\left(M_{\odot} / \mathrm{yr}\right)$ & $\lambda$ & $\log r / r_{\mathrm{S}}$ & $\dot{M} / \dot{M}_{\text {Edd }}$ \\
\hline 17.00 & -11.12 & -2.22 & $1.32 \times 10^{8}$ & $7.49 \times 10^{-3}$ & 0.19 & 3.53 & $3.40 \times 10^{-3}$ \\
16.70 & -10.16 & -1.08 & $2.56 \times 10^{8}$ & $1.74 \times 10^{-2}$ & 0.16 & 3.23 & $7.91 \times 10^{-3}$ \\
16.48 & -9.49 & -0.27 & $4.08 \times 10^{8}$ & $2.90 \times 10^{-2}$ & 0.14 & 3.01 & $1.32 \times 10^{-2}$ \\
16.00 & -8.32 & 1.28 & $9.63 \times 10^{8}$ & $4.77 \times 10^{-2}$ & 0.04 & 2.53 & $2.16 \times 10^{-2}$ \\
15.70 & -8.04 & 1.93 & $1.08 \times 10^{9}$ & $2.30 \times 10^{-2}$ & $\leq 10^{-3}$ & 2.23 & $1.04 \times 10^{-2}$ \\
15.48 & -7.94 & 2.27 & $1.06 \times 10^{9}$ & $1.02 \times 10^{-2}$ & $\leq 10^{-3}$ & 2.00 & $4.64 \times 10^{-3}$ \\
\hline
\end{tabular}

Notation as in Table 1.

accretion flow is hot and optically thin, the thin disk has a "hole".

Also shown in Fig. 1 is a measure for the effective temperature $T_{\text {eff }}^{4}=3 G M \dot{M} / 8 \pi \sigma r^{3}$ near the inner edge of the thin disk. The effective temperature does not scale. The higher the black hole mass the lower is the temperature. These disk temperatures are important for the spectral fits. For comparison we also show the temperature in a disk around a stellar black hole.

The recent investigations of Różańska \& Czerny (2000a, 2000b) mostly concentrate on the hot flow properties and use a two-temperature plasma. They find a slightly different relation between evaporation rate and distance. We investigate the equilibrium where the assumption of a one-temperature plasma is a still valid.

\section{Comparison with observations}

The investigation of nuclei of bright nearby elliptical galaxies by Fabian \& Canizares (1988) already showed that the galactic nuclei are very under luminous. As suggested by Fabian \& Rees (1995) and confirmed by detailed calculations of Mahadevan (1997) the problem is resolved if the galactic nuclei accrete via an ADAF. Likewise the apparently contradictory observations of SgrA* could be understood by advection-dominated accretion (suggestion by Rees 1982, first spectral model by Narayan et al. 1995). Further successful applications of the ADAF model were carried out for the maser galaxy NGC 4258 and the nearby galactic nuclei M 87 and M 60 (for a review see Narayan et al. 1998).
Recent observations in X-rays and in additional different wavelength bands brought further information and at the same time new constraints. Improvement also comes from new measurements of accurate mass and distance, e.g. for NGC 4258 (Herrnstein et al. 1997, 1999).

Di Matteo et al. (1999) examined high-frequency radio observations of three giant low-luminosity elliptical galaxies in the Virgo cluster NGC 4649, NGC 4472 and NGC 4636 using the VLA (Very Large Array) and SCUBA (Submillimetre Common-User Bolometer Array) on the Clerk Maxwell Telescope, and hard X-ray emission. They found radio fluxes lower than the emission predicted by the standard ADAF model. In a second paper Di Matteo et al. (2000) discuss constraints for the advection-dominated flow in the three Virgo ellipticals and three cluster galaxies M 87 (NGC 4486), NGC 1399, and NGC 4696 and suggest that mass loss is important in the radiatively inefficient accretion flow.

In Fig. 2 we summarize the results to show the wide range of values allowed from spectral fits. In investigations including wind loss the quantity $r_{\text {out }}$ is used instead of a transition radius and the wind loss is parametrisized by assuming that the mass flow rate decreases as $\dot{M}=\dot{M}_{\text {out }} \cdot\left(r / r_{\text {out }}\right)^{p}$. For some galaxies only fits for very different truncation radii were investigated, e.g. radii of 300 or $10^{4} r_{\mathrm{S}}$ were considered by Di Matteo et al. (2000) for the elliptical galaxies, together with rates $\dot{M} / \dot{M}_{\mathrm{Edd}}$ in the range 0.01 to 0.001 . We show their best and second best fit. We also show the results from recent ADAF modeling of SgrA* and the very similar galactic transient 
source V404 Cyg (Quartaert \& Narayan 1999) which explains the spectra well without wind loss. Also the observations for M 87 can be modeled without mass loss (Di Matteo et al. 2000). Quataert et al. (1999) pointed out that for accretion rates of $0.03 \dot{M}_{\text {Edd }}$ the thin accretion disks in M 87 and NGC 4696 must be truncated at $\geq 10^{4}$ Schwarzschild radii.

For SgrA*, M 87 (fit without wind loss) and NGC 4649 predictions and fit results agree reasonably. For elliptical galaxies the spectral fits were performed only for radii much larger or much smaller than the transition radius predicted from our model, which makes the comparison difficult. Quataert \& Narayan (1999) argued that infall of gas from a cooling flow could change the location of the truncation. Such a boundary condition is not included in our analysis. But new observations by Chandra might anyhow change the picture. Mushotzky et al. (2000) report on observations of 4 giant elliptical galaxies, NGC 1399, NGC 1401, NGC 4472 and NGC 4636 and the possibility to set upper limits for the radiation, but quantitative results are not yet presented.

For the low luminosity AGN (LLAGN) M 81 and NGC 4579 the observed UV flux demands a truncation far inward, which is in contradiction to our findings. Quataert et al. (1999) already pointed out that it needs to be explained how for similar mass flow rates such different transition radii are found, about $100 r_{\mathrm{S}}$ for the LLAGN and $10^{3}$ to $10^{4} r_{\mathrm{S}}$ for nuclei of elliptical galaxies. These authors raised the question whether the cooling flow in ellipticals could be responsible for this difference. But comparing with our results the LLAGN are the systems which need to be explained. Possibly the evaporation picture can offer an alternative view as will be discussed in the next section. For a review of UV and optical continuum emission in AGN see Koratkar \& Blaes (1999).

\section{Discussion and conclusions}

We discussed how the transition radii predicted by our model compare to the results for the truncation found from the spectral fits using an ADAF for the innermost region and a geometrically thin disk further out. We find three different groups: (1) agreement with our prediction is found for M 87 (model without wind loss) and NGC 4649 (see also Liu et al. 1999). We want to point out the good agreement of our $r-\dot{M}$ relation for the stellar black hole source V404 Cyg, the soft X-ray transient with the best Xray data available. The agreement is generally good in the case of X-ray novae (Meyer et al. 2000b). This is important since the constraints are more severe for the galactic black hole binaries. The observed $\mathrm{H} \alpha$ emission lines give the maximum rotational velocity in the disk and therefore indicates the truncation. (2) The comparison for nuclei of elliptical galaxies is difficult since the radii taken for spectral fits are much larger or smaller than the radii expected from our predictions. A better comparison will be possible when the new Chandra observations (Mushotzky et al. 2000) will become available. (3) A clear discrepancy

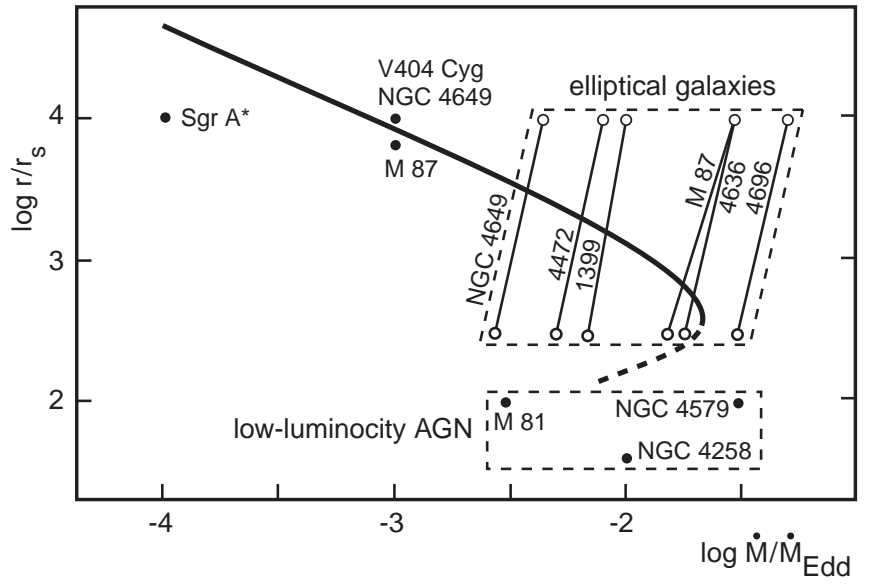

Fig. 2. Comparison of the theoretical $r-\dot{M}$ relation (same as in Fig. 1) with results from fits of observed spectra. Mass accretion rates and radii from various investigations: elliptical galaxies from Di Matteo et al. (2000) (best and second-best fit), low-luminosity AGN M81 and NGC 4579 from Quataert et al. (1999), NGC 4258 from Gammie et al. (1999), Sgr A*, NGC 4649 and the same value for V404 Cyg from Quataert \& Narayan (1999), value for M87 (without wind) from Reynolds et al. (1996). Filled symbols correspond to fits without wind loss, open symbols with wind loss.

is found for the low luminosity AGN (LLAGN), especially M 81.

Concerning the discrepancy found for LLAGN we discuss in the following whether one could imagine other sources of UV radiation than a geometrically thin standard disk. In the modeling the evaporation efficiency has a maximum around a critical radius of $300 r_{\mathrm{S}}$ (the number depends on the chosen value of the viscosity in the hot coronal gas, $\alpha=0.3$, and might be influenced by simplifications in the computational method). If the mass accretion rate is sufficiently low the thin disk should be truncated there or further outward. The decreasing evaporation efficiency inside the critical radius suggests that inside mass could settle again in a thin disk in the equatorial plain. We have not yet included the physics of a two-temperature gas which becomes important with decreasing distance from the black hole. Różańska \& Czerny (2000a) discuss the condensation of matter from the hot coronal flow in a cool disk below. The possible existence of such an interior disk was also mentioned in connection with spectral transitions (Meyer et al. 2000a). We note that such a disk could be cool and the mass flow rate within this disk could be low (an "inert" disk), so that most of the mass flow remains in the ADAF. Spectral observations of a reflection component and the $\mathrm{Fe} \mathrm{K} \alpha$ line, observed for many black hole sources (see e.g. Gilfanov et al. 1999), led to detailed discussions of the accretion flow geometry and give evidence for an inner disk below the ADAF region. In this context it is interesting that Vilhu et al. (2000) modeled observations for the galactic black hole source GRS $1915+105$ with a corona above an interior disk. The interaction of hot protons in the ADAF 
with such an inner disk underneath could transfer accretion energy from the ADAF to the disk where it is radiated away in various wavelength bands, depending on the black hole mass (compare the effective temperatures at the transition radius, Fig. 1). The observed UV radiation then would not necessarily point to a disk truncation at 100 Schwarzschild radii.

Acknowledgements. We thank Friedrich Meyer for discussions.

\section{References}

Abramowicz, M. A., Chen, X., Kato, S., et al. 1995, ApJ, 438, L37

Di Matteo, T., Fabian, A. C., Rees, M. J., et al. 1999, MNRAS, 305,492

Di Matteo, T., Quataert, E., Allen, S. W., et al. 2000, MNRAS, 311,507

Esin, A. A., McClintock, J. E., \& Narayan, R. 1997, ApJ, 489, 865

Fabian, A. C., \& Canizares, C. R. 1988, Nature, 333, 829

Fabian, A. C., \& Rees, M. J. 1995, MNRAS, 277, L5

Gammie, C. F., Narayan, R., \& Blandford, R. 1999, ApJ, 516, 177

Gilfanov, M., Churazov, E., \& Revnivtsev, M. 1999, A\&A, 352, 182

Haardt, F., \& Maraschi, L. 1991, ApJ, 380, L51

Herrnstein, J. R., Moran, J. M., Greenhill, L. J., et al. 1997, BAAS, 191, 2507

Herrnstein, J. R., Moran, J. M., Greenhill, L. J., et al. 1999, Nature, 400, 539

Honma, F. 1996, PASJ, 48, 77

Ichimaru, S. 1977, ApJ, 214, 840

Kato, S., Fukue, J., \& Mineshige, S. 1998, Black Hole Accretion Discs (Kyoto University Press)

Koratkar, A., \& Blaes, O. 1999, PASP, 111, 1

Kusunose, M., \& Mineshige, S. 1994, ApJ, 432, 600
Liang, E. P., \& Price, R. H. 1977, ApJ, 218, 247

Liu, B. F., Yuan, W., Meyer, F., et al. 1999, ApJ, 527, L17

Liu, F. K., Meyer, F., \& Meyer-Hofmeister, E. 1995, A\&A, 300, 823

Mahadevan, R. 1997, ApJ, 477, 585

Meyer, F., \& Meyer-Hofmeister, E. 1994, A\&A, 288, 175

Meyer, F., Liu, B. F., \& Meyer-Hofmeister, E. 2000a, A\&A, 354, L67

Meyer, F., Liu, B. F., \& Meyer-Hofmeister, E. 2000b, A\&A, 361,175

Mushotzky, R. F., Done, C., \& Pounds, K. A. 1993, ARA\&A, 31, 717

Mushotzky, R. F., Angelini, L., Arnaud, K. A., et al. 2000, Bull. AAS 32, HEAD 21.01

Nakamura, K., \& Osaki, Y. 1993, PASJ, 45, 775

Narayan, R., Mahadevan, R., \& Yi, I. 1995, Nature, 374, 623

Narayan, R., Mahadevan, R., \& Quartaert, E. 1998, in The Theory of Black Hole Accretion Discs, ed. M. A. Abramowicz, et al. (Cambridge University Press), 148

Narayan, R., \& Yi, I. 1994, ApJL, 428, 13

Narayan, R., \& Yi, I. 1995a, ApJ, 444, 231

Narayan, R., \& Yi, I. 1995b, ApJ, 452, 710

Quataert, E., Di Matteo, T., Narayan, R., et al. 1999, ApJ, 525, L89

Quataert, E., \& Narayan, R. 1999, ApJ, 520, 298

Rees, M. J. 1982, in The Galactic Center, ed. G. R. Riegler, \& R. D. Blandford, AIP (New York), 166

Rees, M. J., Begelman, M. C., Blandford, R. D., et al. 1982, Nature, 295, 17

Reynolds, C. S., Di Matteo, T., Fabian, A. C., et al. 1996, MNRAS, 283, L111

Różańska, A., \& Czerny, B. 2000a, A\&A, 360, 1170

Różańska, A., \& Czerny, B. 2000b, MNRAS, 316, 473

Svensson, R., \& Zdziarski, A. A. 1994, ApJ, 436, 599

Vilhu, O., Petter, N., Poutanen, J., et al. 2000 [astro-ph0010601]

Życki, P. T., Collin-Souffrin, S., \& Czerny, B. 1995, MNRAS, 277,70 